\title{
Negative Corona Onset Characteristic of the UHV Conductors Based on the Corona Cage
}

\author{
Yun-Peng Liu*, Lei Zhu ${ }^{\dagger}$, Fang-Cheng Lv* and Xiongjie Xie**
}

\begin{abstract}
Negative corona onset characteristics of the stranded conductors based on the ultra-high voltage (UHV) corona cage were studied in the paper. Based on the corona self-sustaining criterion in extreme uneven electric fields and the secondary emission process of the photoelectrons, the corona onset calculation model in the UHV corona cage is established and the corona current tests of the single LGJ900-75, 6 bundle LGJ900-75, 8 bundle LGJ400-35 conductors in dry and rain conditions were done in the UHV corona cage, and the rain rates are $2.4 \mathrm{~mm} / \mathrm{h}, 20 \mathrm{~mm} / \mathrm{h}$ and $30 \mathrm{~mm} / \mathrm{h}$. Corona onset electric field strength is gained by $E-I$ tangent method, and the onset electric field strength in dry condition proves that the calculation model can be used to calculate the corona onset characteristics of the bundle conductors in the UHV corona cage. A further analysis proves that: the negative corona onset voltage of the conductor increases with the bundle number and the diameter of the sub conductor, but decreases with the bundle space in the corona cage. The onset electric field strength is influenced little by bundle space and bundle number, but decreases with the increase of the diameter of the sub-conductor. The surface irregularity coefficient decreases with the rain rate.
\end{abstract}

Keywords: UHV corona cage, Bundle conductor, Charge simulation method, Negative corona onset, Corona current measurement, Rain rate

\section{Introduction}

There are merits like large power capacity, long power transmission distance and saving the power transmission corridors, and many UHV power transmission lines are under construction or some have already been put into operation, due to the rapid development of national economy and power industry. When the surface electric field strength exceeds the breakdown strength of the air, corona discharge occurs and the problems of audible noise, radio interference and corona loss will appear, which will influence the environment and regular operation of the power transmission lines, as in [1-4].

After Peek proposed the well-known Peek formula in 1929, many researchers also proposed the empirical formula to predict the corona onset voltage [5]. Aleksandrov proposed a classic geographic factor that can reflect the photon absorption in the discharge process and analyzed the onset process of the AC conductor [6]. Then the researcher from Egypt studied the corona onset process of the typical rod-plate structure and the smooth conductor and analyzed the influence of temperature on corona onset [7-10]. In [11], the influence of humidity and air pressure

$\dagger$ Corresponding Author: Hebei Provincial Key Laboratory of Power Equipment Security Defense, North China Electric Power University, China. (bao1986dan@163.com)

* Hebei Provincial Key Laboratory of Power Equipment Security Defense, North China Electric Power University, China. (gylyp@ 263.net,1fc0818@sohu.com)

** High Voltage Department, China Electric Power Research Institute, China. (365570141@qq.com.)

Received: June 24, 2013; Accepted: July 19, 2014 on typical point-plate electrode was analyzed.

The surface condition of the conductors will change in the rain, foggy days or in the high humidity weathers, which will reduce the corona onset voltage. In the southern areas of China, it rains quite often and the air humidity is high, which makes it easy for water drops to form on the surface of the conductor and influences the corona characteristics of the power transmission lines. As for the DC corona phenomena, many researchers are studying on radio interference and audible noise, while less research interests are focused on the corona onset characteristics of UHV conductors, it is necessary to study the corona onset characteristic of the UHV bundle conductors both from the theoretical calculation and test statistics as in [12-17].

In this study, each stranded conductor is no longer taken as smooth conductor in the calculation of the onset electric field strength (and voltage), which can better reflect the real electric field condition of the practically used stranded conductor. Based on the gas discharge theory, the corona onset electric field strength of single conductor and bundle conductor are calculated. And the equivalent roughness factors of the conductor in different rain rates are analyzed based on the UHV corona cage.

\section{Materials and Methods}

\subsection{Corona discharge onset process and criterion}

Corona discharge is a sustainable discharge phenomenon 
of the air, which occurs only in part of the air gaps between the polarity, and the space electric field strength between the air gaps is uneven for the practical power transmission lines, the electric filed in the vicinity of the conductor is the strongest, so corona discharge will occur when the electric field strength reaches a certain value. For negative corona discharge, the electrons are sufficiently accelerated that avalanches form in the impact ionization process. Negative ions are formed by combining of electrons to neutral molecules, while the positive ions move toward the conductor. Besides electrons and positive ions, there are also photons in the process, as in [18]. The two main factors that sustain the self-sustaining discharge are that positive ions impacting the cathode and the photoelectron emission in the process of initial avalanche. The radiation energy generated from the impact of positive ions is much higher than the energy of photon radiation, but seldom occurs, thus in this paper, only the photon electron emission from the cathode is considered $[19,20]$.

The impact ionization parameter $\alpha$ and the adhesion coefficient $\eta$ are the function of electric field strength $E$ and air pressure (or relative air density). The free electrons collide with the nitrogen molecule and oxygen molecule, the so called impact ionization occurs:

$$
\begin{aligned}
& e+N_{2} \rightarrow N_{2}^{+}+2 e \\
& e+O_{2} \rightarrow O_{2}^{+}+2 e
\end{aligned}
$$

Suppose that $s=r_{l}$ is the outer radius of the stranded conductor, and $N\left(r_{l}\right)$ is the number of surface electrons of the conductors, then the free electrons in the distance $s=r_{2}$ is :

$$
N\left(r_{2}\right)=N\left(r_{1}\right) \exp \int_{s=r_{1}}^{s=r_{2}}(\alpha-\eta) d s
$$

In the ionization process, the impact ionization coefficient $\alpha$ is larger than the attachment coefficient $\eta$. In the boundary of the corona layer, $\alpha$ equals to $\eta$, so the free electrons stop increasing, and ionization ceases [21].

When the electron avalanches develop continuously, the initial avalanche reach the surface of the conductor and photons will be generated, this is caused by the surface radiation of the initial avalanche, and the formula is as follows:

$$
\begin{gathered}
N_{e p h}=\gamma_{p h} \int_{r_{0}}^{r_{e}} \alpha(r) g(r) \exp \left[-\mu\left(r-r_{0}\right)\right] \cdot \\
\exp \left[\int_{r_{0}}^{r}(\alpha(\xi)-\eta(\xi) d \xi] d r\right.
\end{gathered}
$$

Where $N_{e p h}$ is the number of photons in the discharge process; $\gamma_{p h}$ is the Townsend's second coefficient due to the impact on the cathode by the photons; $g$ is the geometry factor of the effective attachment photons; $\mu$ is the attachment coefficient in the air; $r$ the distance in the space to the center of the conductor; $r_{0}$ is the radius of the conductor; $r_{e}$ is the distance from the boundary to the center of the conductor. $N_{e p h}>1$ is supposed to be the selfsustained discharge condition and the voltage applied corresponding to $N_{e p h}=1$ is considered to be the corona onset voltage. Schumann derived a simple quadratic expression of $\alpha-\eta$, the calculation result of which agrees well with the Paschen's law. The model in this paper is based on the research achievements of Sarma and Janischweskyj, also considering the research results of Mauch, Sander, Harrison and Geballe, the parameters in the formula are referred to [22] and [23].

$$
\begin{gathered}
\frac{\alpha}{\delta}=3632 \exp \left(-168.0 \frac{\delta}{E}\right) \quad 1.9<\frac{E}{\delta}<45.6 \\
\frac{\alpha}{\delta}=7385 \exp \left(-200.8 \frac{\delta}{E}\right) \quad 5.6<\frac{E}{\delta}<182.4 \\
\frac{\eta}{\delta}=9.9865-0.541 \times 10^{-3}\left(\frac{E}{\delta}\right)+1.118 \times 10^{-8}\left(\frac{E}{\delta}\right)^{2}
\end{gathered}
$$

where $\delta=\frac{p}{101.3 \times 10^{3}} \cdot \frac{293}{273+T}$ is the relative air density, $p$ is air pressure $(\mathrm{Pa})$, and $T$ is the temperature in ${ }^{\circ} \mathrm{C}$ during the test.

\subsection{Electric field calculation of the strand conductor in the corona cage}

Charge simulation method (CSM) is one of the calculation methods used for static field numerical calculations. Similar to the image method, based on the uniqueness theorem of the static field, a set of the discrete charges are placed inner the conductor instead of continuous distributed free charges on the surface of polarity (e.g. point charge, line charge or ring charge), which are called simulation charges. The electric field strength can be calculated from the analytical formula of the simulation charge with the superposition theorem, as in [24, 25].

As for a square intersection corona cage, when CSM is applied to calculate the surface electric field of the conductor, each outer strand of the conductor need to be replaced by simulation charges. The number of images of the simulation charges from the cage wall is far larger than from that with no consideration of the outer strand. This will certainly increase the workload and complexity.

The 2D simulation charge model is used to calculate the surface electric field, using the infinite length line charges as the simulation charges, which are placed in the outer strand of the conductor. The outer layer strand is simulated with 8 simulation charges, and the image charge is adopted once to calculate the surface electric field. The simulation charge of the outer stranded conductor and the image charge are shown in Fig. 1. 


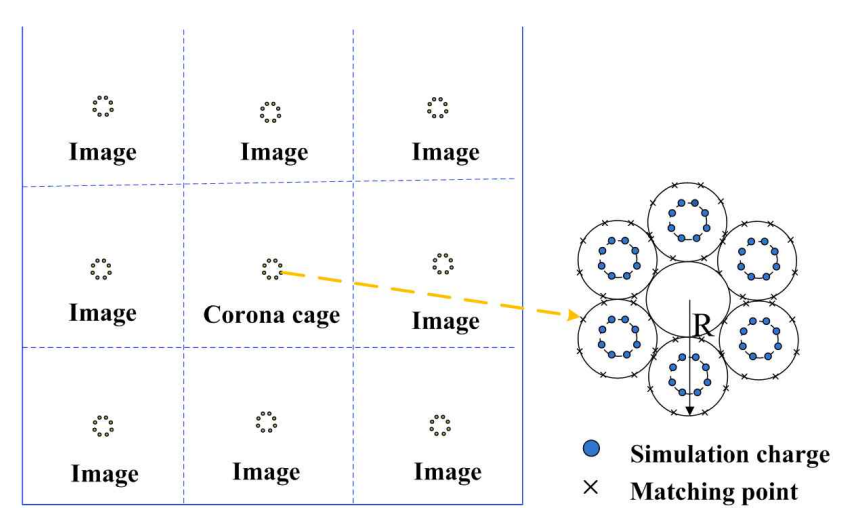

Fig. 1. Bundle conductor simulation charge and image charge distribution

The calculation formula of the potential coefficients $P$ cage, $P$ mirror and electric field strength coefficients

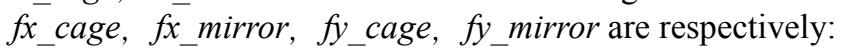

$$
\begin{gathered}
P_{-} \text {cage }=-(1 / 2 \pi \varepsilon) \ln \left(\rho_{1}\right) \\
P_{-} \text {mirror }=-(1 / 2 \pi \varepsilon) \ln \left(\rho_{2}\right) \\
f x_{-} \text {cage }=(1 / 2 \pi \varepsilon)\left(x-x_{1}^{\prime}\right) / \rho_{1}{ }^{2} \\
f x_{-} \text {mirror }=(1 / 2 \pi \varepsilon)\left(x-x_{2}{ }^{\prime}\right) / \rho_{2}{ }^{2} \\
f y_{-} \text {cage }=(1 / 2 \pi \varepsilon)\left(y-y_{1}\right) / \rho_{1}{ }^{2} \\
f y \text { cage }=(1 / 2 \pi \varepsilon)\left(y-y_{2}{ }^{\prime}\right) / \rho_{2}{ }^{2}
\end{gathered}
$$

where $\rho_{1}=\sqrt{\left(x-x_{1}^{\prime}\right)^{2}+\left(y-y_{1}^{\prime}\right)^{2}}$,

$$
\rho_{2}=\sqrt{\left(x-x_{2}^{\prime}\right)^{2}+\left(y-y_{2}^{\prime}\right)^{2}}
$$

$\rho_{1}$ and $\rho_{2}$ are respectively the distances of simulation charge $\tau\left(x_{1}^{\prime}, y_{1}^{\prime}\right)$ and its image line charge $\tau\left(x_{2}^{\prime}, y_{2}^{\prime}\right)$ to the field point $(x, y)$, and $\tau=-\tau$ '.

8 bundle LGJ-400-35 (bundle space $400 \mathrm{~mm}$ ) is taken for example. The number of outer strands of LGJ-400-35 is 22 . For the corona cage of square cross-section $8 * 8 \mathrm{~m}$, when the calculation voltage is $100 \mathrm{kV}$, the space electric field strength around the sub conductor surface was

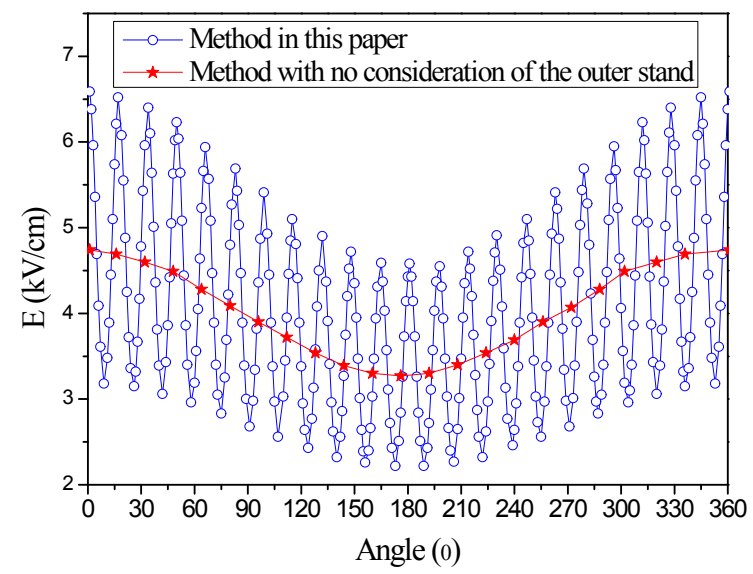

Fig. 2. Surface electric field of the sub conductor

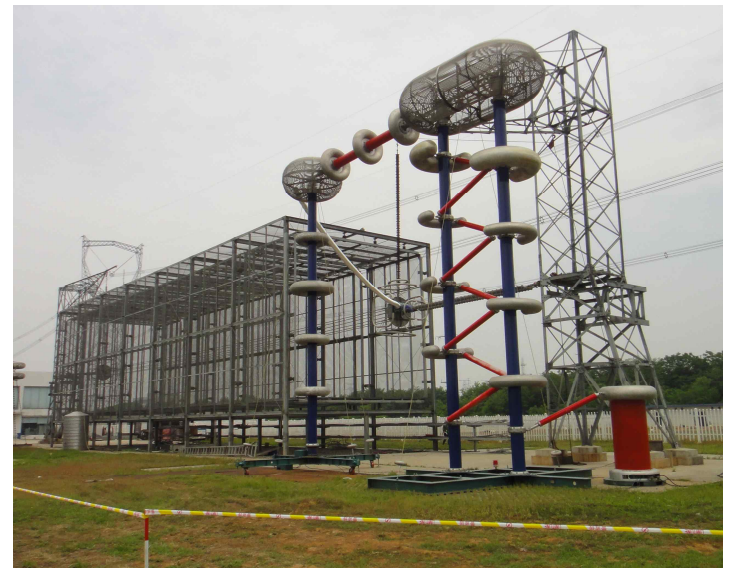

Fig. 3. UHV corona cage

calculated and compared with the method with no consideration of the outer strand.

And the calculation result is shown in Fig. 2. Peak points appear outside every outer strand. And 22 peak values appear in Fig. 2. As shown in the figure that compared to the methods with no consideration of the outer stand, there are extreme values appear at each single strand, which is consistent with the practical situation. So the charge simulation method for the stranded conductors can be used to calculate the space electric-field strength around conductors in the corona cage. Therefore, a more accurate corona onset calculation model can be established.

\subsection{UHV corona cage}

The UHV corona cage in the UHV test base in Wuhan, China is square in cross section; the side length of the cross section is $8 \mathrm{~m}$, the measurement section is $25 \mathrm{~m}$ long, and the protection section is $5 \mathrm{~m}$ long. The UHV corona cage is equipped with an artificial rain system, and the rain rates from $2.4 \mathrm{~mm} / \mathrm{h}$ to $30 \mathrm{~mm} / \mathrm{h}$ could be simulated. UHV corona cage is shown in Fig. 3.

\subsection{DC corona current measurement method}

The schematic diagram and the figure of the corona current measurement equipment based on GPS clock are shown in Fig. 4 and Fig. 5. 1-3 are the segments of the UHV corona cage. The no inductive resistor $4(1 \mathrm{~K} \Omega)$ is used to convert the DC current signal into voltage signal. A sampling card is equipped in the upper computer 5 and the lower computer 15 respectively, with the maximum sampling rate up to $120 \mathrm{MS} / \mathrm{s}$. The function of the upper computer 5 is to control the sampling of the current signal and data transmission with the lower computer. The synchronous measure of the voltage and current signal is realized by the use of GPS synchronous clock 6 and 9 . The trigger time is 1 minute in this test, which means the trigger signal will be sent out each minute. The time error between the two GPS clocks is less than $1 \mu \mathrm{s}$. The 


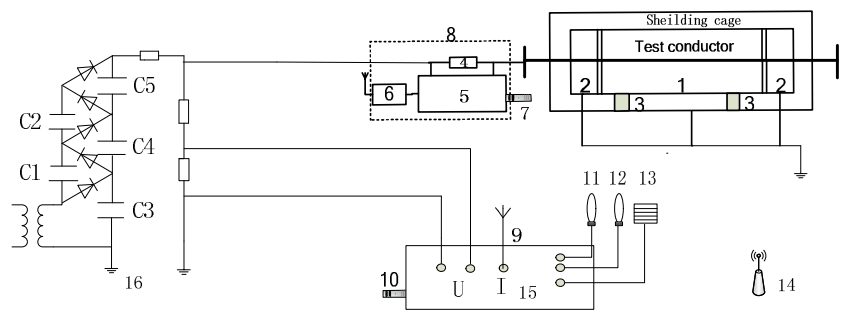

Fig. 4. Sketch of the measurement system

1-corona cage measurement segment; 2-protection segment; 3insulation support; 4-sampling resistor; 5-upper computer; 6upper GPS; 7-wireless net card; 8-Faraday cage; 9lower GPS; 10-wireless net card; 11-temperature sensor; 12humidity sensor; 13-pressure sensor; 14-wireless router; 15-lo wer computer; 16-DC UHV generator

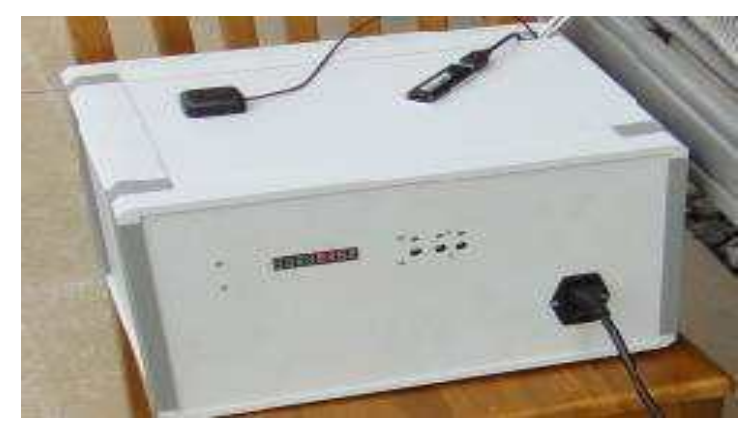

Fig. 5. GPS upper computer

wireless net cards 7 and 10 and the wireless router 14 compose the wireless network for data transmission between the upper computer and the lower computer, which can separate the high voltage side and the low voltage side, so there is no electric connection between them. The maximum data transmission rate of the network is $150 \mathrm{MS} / \mathrm{s}$. During the corona current measurement tests, the environmental temperature, humidity, and air pressure are recorded by 11,12 , and 13 . The type of the UHV DC voltage generator 16 is ZGF-1200/10, the rated voltage is $1200 \mathrm{kV}$, which can satisfy the requirement of the DC corona test.

The software system is programmed with the software Labview. Here is how the system works: after receiving the trigger signals from 6 and 9, the upper computer and lower computer work synchronously. The upper computer5 samples the current signal and transmits it to the lower computer through the wireless network. The lower computer 15 samples the voltage signal and records both the current signal and voltage signal.

\section{Results and Discussion}

\subsection{Corona current measurement result}

The corona current curves of single conductor LGJ90075, six bundle LGJ900-75 (bundle space $450 \mathrm{~mm}$ ) and
Table 1. Operating conditions during the tests

\begin{tabular}{c|c|c|c}
\hline Conductor type & $\begin{array}{c}\text { Temperature } \\
\left({ }^{0} \mathrm{C}\right)\end{array}$ & $\begin{array}{c}\text { Relative humidity } \\
(\%)\end{array}$ & $\begin{array}{c}\text { Air pressure } \\
(\mathrm{kPa})\end{array}$ \\
\hline Single LGJ900-75 & $20.9-25.2$ & $49.6-60.4$ & $101.6-101.7$ \\
\hline 6 bundle LGJ900-75 & $22.6-26.8$ & $50.9-58.6$ & $101.3-101.5$ \\
\hline 8 bundle LGJ400-35 & $18.9-24.6$ & $40.2-50.8$ & $101.8-102$ \\
\hline
\end{tabular}

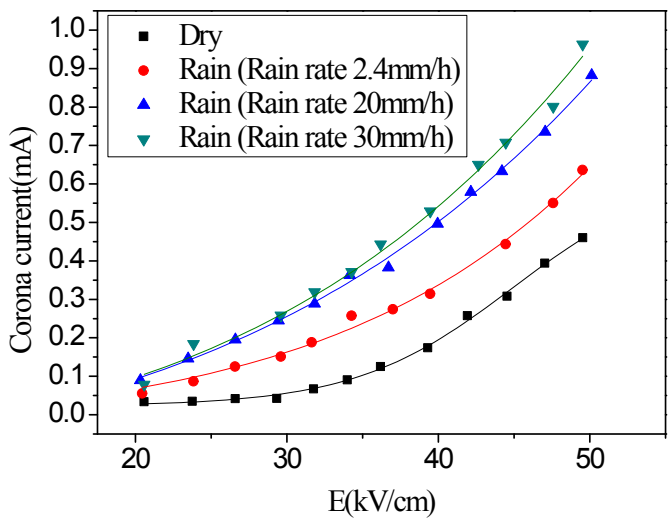

Fig. 6. Corona currents of the single LGJ900-75 under DC negative voltage in different rain rate

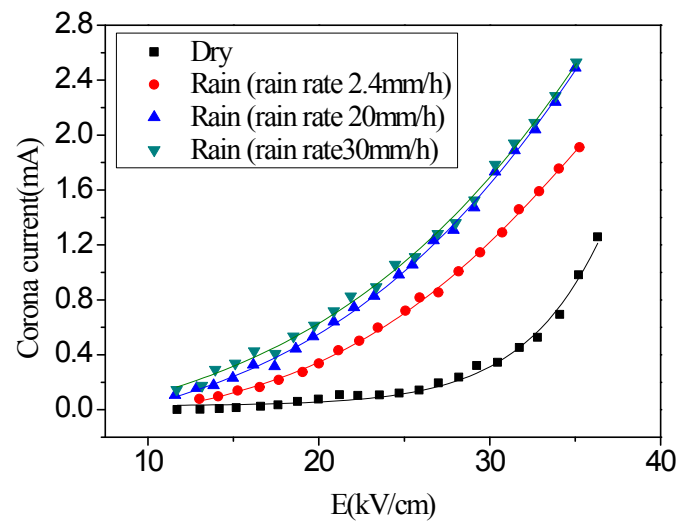

Fig. 7. Corona currents of the six bundle LGJ900-75 under DC negative voltage in different rain rates

eight bundle LGJ400-35 (bundle space $400 \mathrm{~mm}$ ) in fine and rain conditions are shown in Figs. 6-Fig. 8, the rain rate are $2.4 \mathrm{~mm} / \mathrm{h}, 20 \mathrm{~mm} / \mathrm{h}$ and $30 \mathrm{~mm} / \mathrm{h}$. The temperature, relative humidity and air pressure during the DC corona current measurement are shown in Table 1. The diameters of the LGJ400-35 and LGJ900-75 are $26.82 \mathrm{~mm}$ and $40.7 \mathrm{~mm}$ respectively. The abscissa is the maximum electric field strength with the consideration of the outer strand. As could be seen in the test results, the corona current is larger in simulated rain than in dry condition, which increases rapidly from dry condition (rain rate 0$)$ to light rain $(2.4$ $\mathrm{mm} / \mathrm{h}$ ), while increase slightly from rain rate $20 \mathrm{~mm} / \mathrm{h}$ to rain rate $30 \mathrm{~mm} / \mathrm{h}$. For example, when the electric filed strength is $35 \mathrm{kV} / \mathrm{cm}$, corona currents of eight bundle LGJ400-35 conductor corresponding to the rain rate 0 $\mathrm{mm} / \mathrm{h}, 2.4 \mathrm{~mm} / \mathrm{h}, 20 \mathrm{~mm} / \mathrm{h}$ and $30 \mathrm{~mm} / \mathrm{h}$ are $0.28 \mathrm{~mA}, 1.31$ $\mathrm{mA}, 1.8 \mathrm{~mA}$, and $1.91 \mathrm{~mA}$. With the increase of the rain 


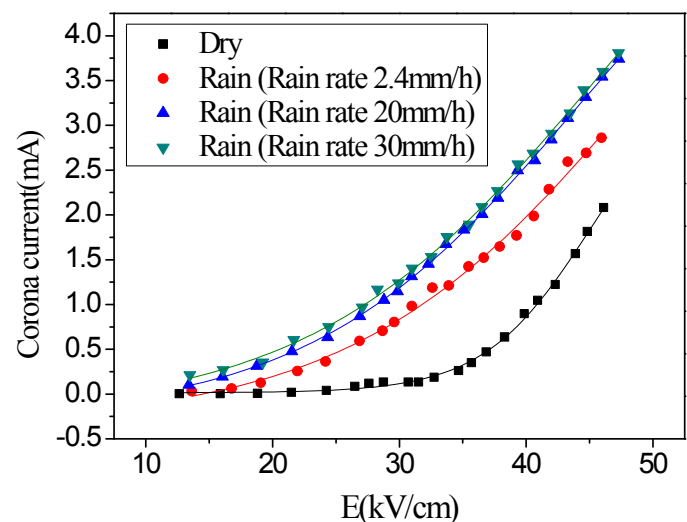

Fig. 8. Corona currents of the eight bundle LGJ400-35 under DC negative voltage in different rain rates

rate, the corona current has reached the saturation state.

\subsection{Judgment of corona onset in the test}

There are many ways to judge the corona onset electric field strength (or voltage) in the practical test: UV imaging, current impulse method from the corona current, third harmonic method of corona current, corona current parameter method, and E-I curve fitting method, etc, the way of most method is: firstly, search for the characteristic parameter of corona onset, then gain the variation curves of these parameters with the test electric field strength and finally determine the inflection point of the curve, which is considered as the corona onset point. Among these judgment methods, the tangent line method is simple and convenient to use, and is largely applied in the practical project. The principle of tangent line method is in Fig. 9, that is drawing the tangent line before and after corona onset, the intersection point of the two lines is the corona

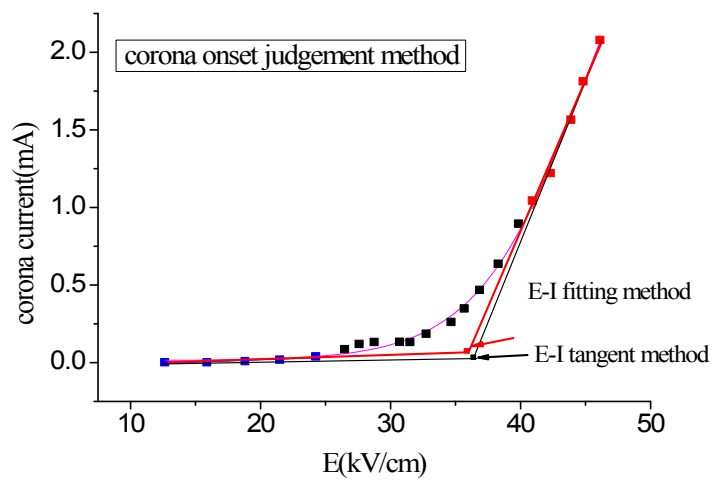

Fig. 9. Corona onset judgment method

Table 2. Corona onset electric filed strength with different methods

\begin{tabular}{c|c|c}
\hline \multirow{2}{*}{} & \multicolumn{2}{|c}{ Onset electric field strength $E(\mathrm{kV} / \mathrm{cm})$} \\
\cline { 2 - 3 } & Fine weather & Rain $(30 \mathrm{~mm} / \mathrm{h})$ \\
\hline E-I tangent & 36.04 & 28.63 \\
\hline E-I fitting & 36.19 & 28.89 \\
\hline Third harmonic wave & 36.29 & 28.66 \\
\hline
\end{tabular}

onset point. The two methods with curve fitting method and corona current tangent method are compared in Fig. 9.

The corona onset electric field strengths with three commonly used methods are compared in Table 2. And the comparison of tangent method and corona pulse method are shown in [25], there are with a maximum error less than $2 \%$ in that paper. So the corona onset electric field strength with corona tangent method can be compared to the other corona onset judgment methods.

\subsection{Calculation and test result of onset electric field strength in dry condition}

The corona onset electric field strength of the three kinds of conductors (single conductor LGJ900-75, 6 bundle LGJ900-75 and 8 bundle LGJ400-35) in dry conditions are calculated with the corona onset model of the conductor in the UHV corona cage, as Table 3 shows. As could be seen in the table that the calculation results of the 6 bundle conductor and 8 bundle conductor agree well with the test results, while the differences of the single conductor between the calculation and measured result is a little bit higher, which could be explained as: although the conductors are purchased in the same time, there are still differences between the individual conductor, the surface condition of the single conductor may be better than the other conductors, the contamination, ware or the burrs are less than the average level. To sum up, it could be concluded that the calculation method mentioned in this paper could be used in the calculation of the negative corona onset voltage of the bundle conductors in the UHV corona cage, and the calculation result is correct and reliable.

Table 3. Comparison of the corona onset electric field

\begin{tabular}{c|c|c|c}
\hline Conductor form & $\begin{array}{c}\text { Test } \\
(\mathrm{kV} / \mathrm{cm})\end{array}$ & $\begin{array}{c}\text { Calculation } \\
(\mathrm{kV} / \mathrm{cm})\end{array}$ & $\begin{array}{c}\text { error } \\
(\%)\end{array}$ \\
\hline Single LGJ900-75 & 35.03 & 33.26 & $5 \%$ \\
\hline 6 bundle LGJ900-75 & 32.19 & 33.23 & $-3.9 \%$ \\
\hline 8 bundle LGJ400-35 & 36.19 & 35.16 & $2.8 \%$ \\
\hline
\end{tabular}

\subsection{The influence of structural parameters to corona onset characteristics}

The influence of structural parameters of UHV conductors on corona onset characteristics is analyzed in three aspects: bundle number, bundle space and diameter of sub conductor. Strand conductors LGJ900-75 (bundle space $450 \mathrm{~mm}$ ) and LGJ400-35 (bundle space $400 \mathrm{~mm}$ ) of different bundle number, varying from single conductor to eight bundle conductors, are calculated. Fig. 10 shows that the corona onset voltage increases with the increase of bundle number. But the increase of the corona onset voltage with the bundle number tends to be saturated. The onset electric field strength is almost unchanged with the increase of bundle number, as shown in Fig. 11.

The corona onset voltage decreases with the increase of 
the bundle space, as shows in Fig. 12, and the calculated bundle spaces are from $350 \mathrm{~mm}$ to $500 \mathrm{~mm}$, which are the regular lengths of the practical use bundle conductors. The onset electric field strength is almost unchanged with the increase of bundle space, as shown in Fig.13.

The variation curve of the corona onset voltage of 6 bundle conductor (bundle space $450 \mathrm{~mm}$ ) and 8 bundle conductor (bundle space $400 \mathrm{~mm}$ ) with the variation of the diameter of sub conductors are shown in Fig.14, which is obviously that the increase of diameter of sub conductor

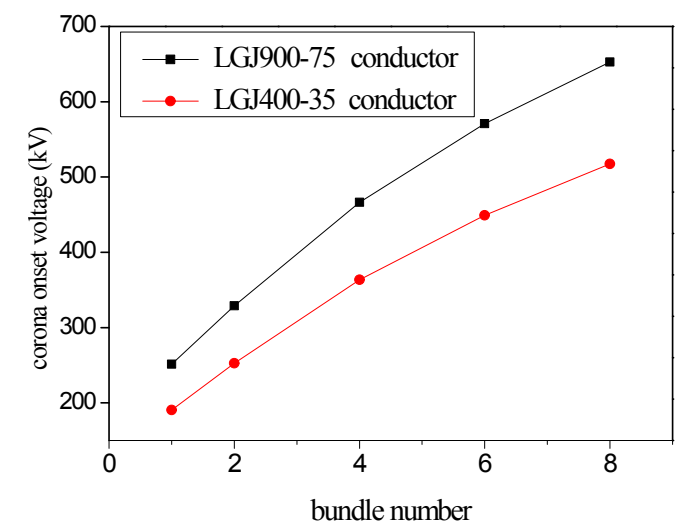

Fig. 10. Variation of the onset voltage with bundle number

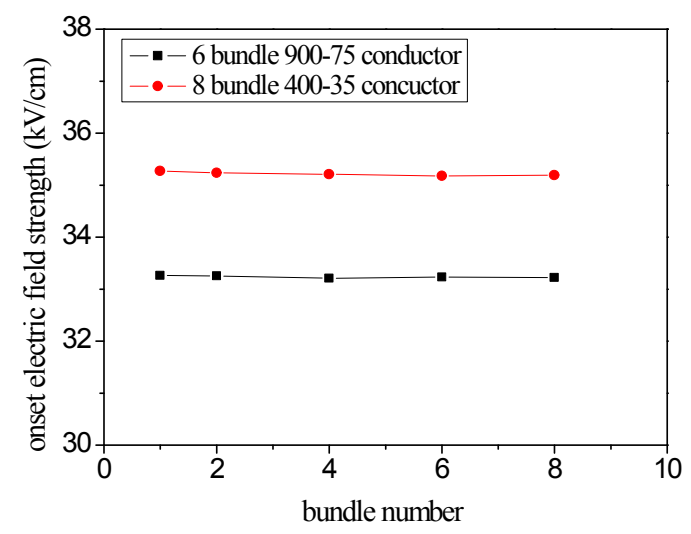

Fig. 11. Variation of the corona onset electric field strength with bundle number

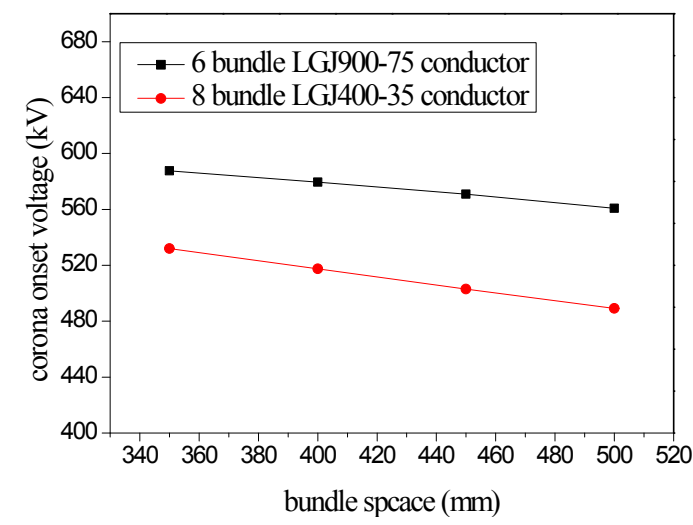

Fig. 12. Variation of corona onset voltage with the bundle space could also improve the corona onset characteristics of the bundle conductor, but there is also saturation phenomena. The corona onset electric field strength decreases with the increase of sub conductor diameters, as shown in Fig. 15.

The power transmission line is designed to meet a certain Radio Interference (RI) level, which in turn limits corona loss to a reasonable level. The above figures and related data are used to determine the structural parameters that would meet the RI (and corona loss) design target. And some other factors, just like vibration of the conductor

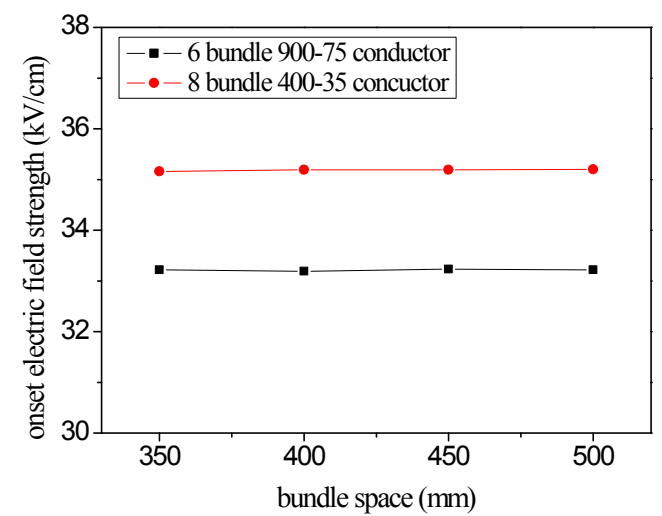

Fig. 13. Variation of the corona onset electric field strength with bundle space

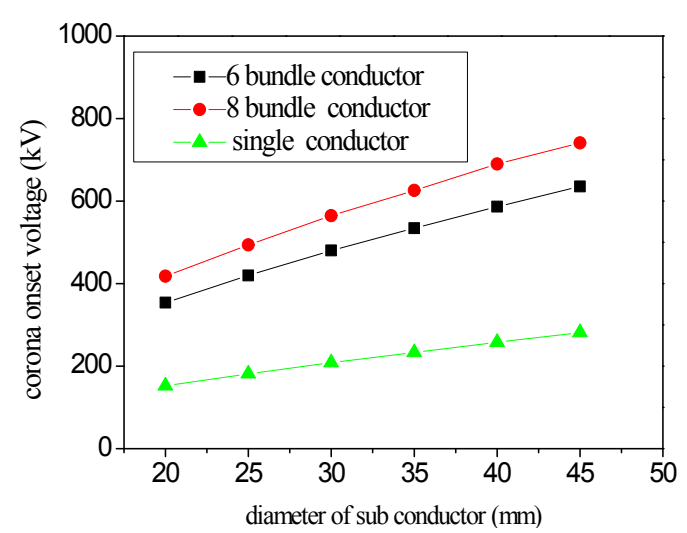

Fig. 14. Variation of the onset voltage with the diameter of sub-conductor

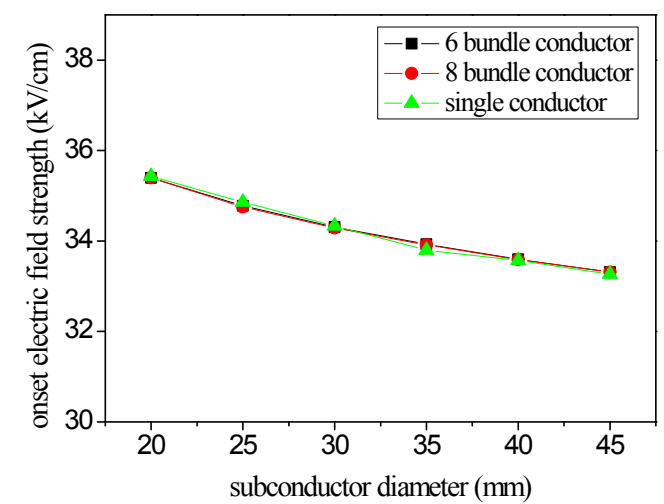

Fig. 15. Variation of the onset electric field strength with the diameter of sub-conductor 
should also be considered. So the above mentioned figures and relative data, the bundle number, bundle space and sub conductor are from the corona characteristics of the transmission line conductors aspects and should be determined comprehensively together with the economic aspect in the practical engineering.

\subsection{Analysis of the surface irregularity factor of the conductors in rain condition}

The environmental and weather condition show significant influence on corona characteristics. In the combined effect of gravity, surface tension and electric field force, a tip will form in the rain droplet on the surface of the conductor, which will split into several small droplets and fall, and then new droplets will form in the surface of the conductor. The existence of the rain drop greatly influences the surface electric field strength of the conductor. The surface irregularity coefficient is the ratio of the corona onset test voltage (electric field strength) in the rain condition to the corresponding value of smooth conductor in the dry condition. So the increase of the surface irregularity will decrease the surface irregularity coefficient.

Table 4 shows the influence of rain rate on the surface irregularity coefficient, and the whole tendency is similar: the surface irregularity coefficient decreases with the increase of the rain rate, while eventually tends to be saturated. And for the bundle conductor the surface irregularity factor $\mathrm{m}$ is similar to the value in [3], in that paper the irregularity factor $\mathrm{m}$ varies from 0.75 in the very light rain to about 0.5 in the heavy rain for bundle conductors.

Here is the possible explanation: when the rain is light, with the increase of the rain rate, the droplet hanging on the conductor also increase, which will distort the surface electric field strength of the conductor and corona discharge occurs on a lower voltage, but continuing increasing the rain rate will lead to saturation of the rain droplets, that the conductor is fully hanging with rain droplet and there is no extra position for the new droplets, the falling droplets will be replaced rapidly with the new ones, and keeping increasing the rain rate is so limited in reducing the surface irregularity coefficient.

Table 4. Corresponding surface irregularity coefficient of the conductor with rain rate

\begin{tabular}{c|c|c|c}
\hline \multirow{2}{*}{$\begin{array}{c}\text { Rain rate } \\
(\mathrm{mm} / \mathrm{h})\end{array}$} & \multicolumn{3}{|c}{ Surface irregularity coefficient } \\
\cline { 2 - 4 } & Single conductor & Six bundle & Eight bundle \\
\hline 2.4 & 0.64 & 0.56 & 0.56 \\
\hline 20 & 0.59 & 0.48 & 0.49 \\
\hline 30 & 0.59 & 0.47 & 0.48 \\
\hline
\end{tabular}

\section{Conclusion}

1) A calculation model of the negative corona onset electric field strength (and voltage) of the bundle conductors in the UHV corona cage is established, in which the influence of outer strands on the surface electric field, the secondary photons emission and corona onset judgment in the extremely non-uniform electric field are taken into consideration. The outer layers of stranded conductors are simulated with 8 simulation charge and one time image is used in surface electric field strength calculation.

2) The corona current tests of single conductor LGJ900-75, 6 bundle LGJ900-75(bundle space $450 \mathrm{~mm}$ ), 8 bundle LGJ400-35(bundle space $400 \mathrm{~mm}$ ) in dry and rain conditions were done in the UHV corona cage in the UHV Test Base of the State Grid and the corona onset voltage is gained with the tangent line method. After the comparison of calculated values and test values in dry condition, a conclusion could be made that the calculation model mentioned in this paper could be applied in calculation of negative corona onset electric field strength (and voltage) in the UHV corona cage.

3) In the UHV corona cage, the corona onset voltage increases with the bundle number, while there is saturation tendency. In the bundle space range of between $350 \mathrm{~mm}$ to $500 \mathrm{~mm}$, the corona onset voltage of the conductor decreases with the increase of the bundle space. With the bundle number and bundle space fixed, the corona onset voltage increases with the increase of sub conductor and the increase tends to be saturated. The onset electric field strength is almost unchanged with the increase of bundle space and bundle numbers. The corona onset electric field strength decreases with the increase of the sub conductor diameters.

4) In the rain condition, the existing of rain droplets increases the surface irregularity and the calculated surface irregularity coefficient decreases with the increase of the rain rate, but when the rain rate increases to certain value, the increase of the surface irregularity tends to be saturated.

\section{Acknowledgements}

This work was supported by the following projects: National Basic Research Program of China (2011CB 209401), National Natural Science Foundation of China (51277073) and Technical project of the Stage Grid of China(SG0955).

\section{References}

[1] Y. B. Shu, W. L. Zhang, "Research of Key Technologies for UHV Transmission", Proceedings of the CSEE, vol. 27, no. 31, pp.1-6, Nov. 2007. (in Chinese)

[2] Y. B. Shu, Y. Hu, "Research and application of the key technologies of UHV AC transmission line", Proceedings of the CSEE, vol. 27, no. 36, pp. 1-7, 
Dec. 2007. (in Chinese)

[3] J. J. Clade, C. H. Gary, "Predetermination of corona losses under rain: experimental interpreting and checking of a method to calculate corona losses", IEEE Trans. Power Apparatus and System, vol. 89, no. 5, pp. 853-860, May. 1970.

[4] Y. P. Liu, S. H. You, Q. F. Wan, W. J. Chen, F. C. Lv, Y. Chen, "Research on UHV AC Single Circuit Test Line Corona Losses under Rain", Proceedings of the CSEE, vol. 30, no. 19, pp. 114-119, Jul. 2010. (in Chinese)

[5] H. Gildas, "Theoretical evaluation of Peek's law", IEEE Trans. Industry Application, vol. 20, no. 6, pp. 1647-1651, Nov 1984.

[6] G. N. Aleksandrov, "Physical conditions for the foundation of an alternating current corona discharge", Soviet Physics-Technical Physics, vol. 1, no. 8, pp. 1714-1736, Aug 1956.

[7] M. Sarma and W. Janischewskyj, "DC corona on smooth conductors in air", Proceedings of the Institutions of Electrical Engineering, vol. 116, no. 1, pp. 161-166, Jan 1969.

[8] M. Abdel-Salam and D. Wiitnam, "Calculation of corona onset voltage for duct-type precipitators", IEEE Trans. Industry Application, vol. 29, no. 2, pp. 274-280, Mar. 1993.

[9] M. M. EI-Bahy, M Abouelsaad, "Onset voltage of negative corona on stranded conductors", Journal of Physics D: Applied Physics, vol. 40, no. 10, pp. 3094-3101, May. 1970.

[10] M. Abdel-Salam, "Calculating the effect of high temperatures on the onset voltages of negative discharges", Journal of Applied Physics, vol. 9, no. 12, pp. 149-154, Aug. 1976.

[11] X. M. Bian, J. F. Hui, H. K. Huang, L. M. Wang, Z. C .Guan, Y. Chen, "Research on negative DC corona characteristics related to air pressure and humidity", Proceedings of the CSEE, vol. 30, no. 4, pp. 118-124, Apr. 2010. (in Chinese)

[12] Q. L. Wang, L. Zhang, L. Zhou, B. Z. Ren, J. B. Fan, C. Gu, Q. G. Zhang, "Influence of corona characteristics of water drops on smooth conductor under negative DC voltage", High Voltage Apparatus, vol. 47, no. 8, pp. 9-17, Aug. 2011. (in Chinese)

[13] M. H. Ma, Research on corona of single slick conductor and contaminated conductor in a small cage, Master thesis, Department of Electric Engineering: Tsinghua University, pp. 1-3, 2007, China. (in Chinese)

[14] Y. M. Zhao, M. H. Ma, J. W. Chu, Z. C. Guan, L. M. Wang, "Measurement methods for DC conductor corona onset voltage in the corona cage", High Voltage Engineering, vol.34, no. 8, pp. 1567-1572, Aug. 2008. (in Chinese)

[15] P. S. Maruvada., Corona performance of high-voltage transmission lines, London, UK: Research Studies Press Ltd, 2000, pp. 65-66.

[16] J.B. Fan, Z.X. Li, C. Gu, Y. Yu, Z.Y. Su, “Calculation method for DC onset corona voltage", Transactions of China Electro-technical Society, vol. 23, no. 10, pp. 100-105, Oct 2008. (in Chinese)

[17] J. J. Yang, Gas discharge, Beijing: Science Press, 1983, pp. 20-25. (in Chinese)

[18] Z. Ali, G. Ahamad, Z. Vahraz, "A new method for calculation of corona inception voltage in stranded conductors of overhead transmission lines", in Proc. 2006 First International Power and Energy Conference, Putra Jaya, Malaysia, Nov. 2006.

[19] R. Lin, "Research on DC positive corona discharge characteristics of conductors and its affecting factors", Master thesis, Department of Electric Engineering, Chongqing University, pp. 23-26, 2009, China. (in Chinese)

[20] W. Li, B. Zhang, J.L. He, R. Zeng, X.L. Li, Q. Wang, "Corona losses calculation of UHV/EHV AC transmission lines", Proceedings of the CSEE, vol. 29, no. 19, pp. 118-124, Jul 2009. (in Chinese)

[21] F. C. LÜ, S. H. You, Y. P. Liu, Q. F. Wan, Z. B. Zhao, "AC Conductors' Corona-loss Calculation and analysis," IEEE Trans. Power Delivery, vol. 27, no. 2, pp. 877-885, Apr 2012

[22] Y. S. Zheng, J. L. He, B. Zhang, R. Zeng, Z. Q. Yu. "Photoemission replenishment criterion for inception of negative discharge in air", IEEE Trans. Power Delivery, vol. 14, no. 4, pp. 46-50, July. 2011.

[23] L. Gong, "Study on corona onset characteristics influenced by conductor surface status under low air pressure", Master thesis, Department of Electric Engineering, Chongqing University, pp. 19-24, 2007 China. (in Chinese)

[24] S. Y. Xie, C. Liu, Z. Li, "The method for measuring initial corona of super high voltage transmission line", High Voltage Apparatus, vol. 25, no. 6, pp. 39-41, Apr. 1989. (in Chinese)

[25] X. M. Bian, D. M. Yu, Chen L, J. M. K. MacAlpine, L. M. Wang, and Z. C. Guan, "Influence of aged conductor surface conditions on AC corona discharge with a corona cage. IEEE Tran. Dielectrics and Electrical Insulation", vol. 18, no. 3, pp. 809-817, Jan. 2011.

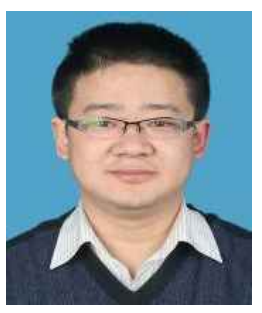

Yun-peng Liu He received the B. Sc degree in Electrical Engineering from North China Electric Power University, Baoding, China, in 1999, and $\mathrm{Ph}$. D. degree from the same university in 2005. He is a doctoral supervisor and a professor in North China Electric Power University, Baoding, China. His research interests are UHV transmission, and fault detection and diagnosis of electric equipment. 


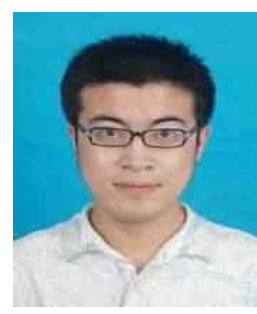

Lei Zhu He received the B. Sc degree from North China Electric Power University, Baoding, China, in 2008. He is a doctor candidate of North China Electric Power University, Baoding, China. His research interests are corona discharge, and fault detection and diagnosis of electric equipment

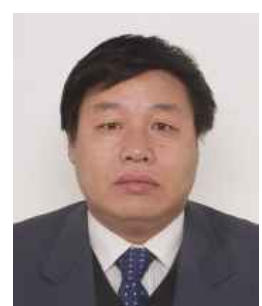

Fang-cheng Lv He received the Ph.D. degree in Electrical Engineering from North China Electric Power University, Baoding, China in 2000. He is a Professor, Doctoral Supervisor in North China Electric Power University, Baoding, China. His research interests are electrical equipment insulation mechanism, fault detection and diagnosis of electric equipment.

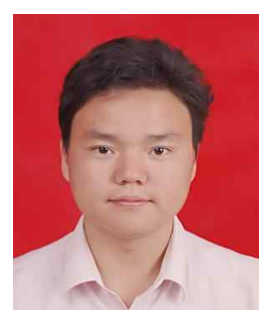

Xiong-jie Xie was born in 1983 . He received the B.Sc degree in Electrical Engineering from Wuhan University, Wuhan, China, in 2005, and MEng degree from the same university in 2007. $\mathrm{He}$ is now a doctor candidate. His research interest are high voltage test technology, oil-SF6 immersed bushing and corona characteristics of the power transmission equipments. 